\title{
Monolayer fluoro-InSe: Formation of a thin monolayer via fluorination of InSe
}

\author{
M. Yagmurcukardes* \\ Department of Physics, University of Antwerp, Groenenborgerlaan 171, B-2020 Antwerp, Belgium
}

(Received 20 May 2019; revised manuscript received 18 June 2019; published 24 July 2019)

\begin{abstract}
By performing density functional theory-based first-principles calculations, the formation of a thin monolayer structure, namely InSeF, via fluorination of monolayer InSe is predicted. It is shown that strong interaction of F and In atoms leads to the detachment of In-Se layers in monolayer InSe and 1T-like monolayer InSeF structure is formed. Monolayer InSeF is found to be dynamically stable in terms of its phonon band dispersions. In addition, its Raman spectrum is shown to exhibit totally distinctive features as compared to monolayer InSe. The electronic band dispersions reveal that monolayer InSeF is a direct gap semiconductor whose valence and conduction band edges reside at the $\Gamma$ point. Moreover, the orientation-dependent linear elastic properties of monolayer InSeF are investigated in terms of the in-plane stiffness and Poisson ratio. It is found that monolayer InSeF displays strong in-plane anisotropy in elastic constants and it is slightly softer material as compared to monolayer InSe. Overall, it is proposed that a thin, direct gap semiconducting monolayer $\mathrm{InSeF}$ can be formed by full fluorination of monolayer InSe as a new member of the two-dimensional family.
\end{abstract}

DOI: 10.1103/PhysRevB.100.024108

\section{INTRODUCTION}

Dimensional reduction of layered materials from their bulk form to a two-dimensional (2D) structure significantly influence their electronic, dynamical, and optical properties. Following the synthesis of graphene [1], many other layered bulk materials successfully transformed into their $2 \mathrm{D}$ monolayer forms. Recently, monolayers of group-III monochalcogenides, GaS, GaSe, GaTe, and InSe, have been successfully added to the library of the 2D family [2-6]. In this class of layered materials, especially the fundamental properties of Ga-monochalcogenides were widely investigated and reported [7-17].

Indium selenides, members of the group-III chalcogenides family, can take a wide variety of structural forms, such as $\mathrm{In}_{2} \mathrm{Se}_{3}$ and $\mathrm{InSe}$, in different crystal structures such as rhombohedral and hexagonal phases. $\mathrm{In}_{2} \mathrm{Se}_{3}$ was reported to crystallize in rhombohedral crystal symmetry with three different phases ( $\alpha, \beta$, and $\gamma$ phases) which can transform from one to another under ambient conditions [18-22]. The monochalcogenide form of indium selenide (InSe), however, exists in a layered hexagonal structure in nature. Following its isolation to the monolayer form [6], its bulk and fewlayer properties were widely investigated [23-29]. Bringuier et al. studied the optical properties of bulk InSe and showed that it exhibits large optical nonlinearities [30]. In another study, Jones et al. investigated the optical nonlinearities in both the bulk and surface of InSe and observed a quadratic increase in the second harmonic generation signal for thinner crystals [31]. Recently, Lei et al. investigated the evolution of electronic band structure of few-layer InSe and reported that due to the suppressed interlayer electron orbital coupling, the band gap increases as going from bulk to few-layer thickness

\footnotetext{
*Mehmet.Yagmurcukardes@uantwerpen.be
}

[32]. Very recently, Bandurin et al. successfully isolated the 2D monolayer form of InSe and demonstrated its device performance from few layer to monolayer [6]. In addition, by photoluminescence (PL) spectroscopy measurements it was reported that a direct-to-indirect band gap transition can be observed from bulk to monolayer InSe. To clarify the correct number of layers for such transition, theoretical studies were also achieved. Mudd et al. showed both theoretically and experimentally that after the thickness of 20-layer InSe, a PL intensity can be observed for the band gap measurements [33].

Functionalization of $2 \mathrm{D}$ materials by different types of adatoms such as $\mathrm{H}, \mathrm{F}, \mathrm{Cl}$, or $\mathrm{Li}$, was both experimentally and theoretically investigated [34]. Among these atoms, the $\mathrm{F}$ atom with its relatively high electronegativity was considered for the functionalization of graphene. First, effect of fluorination on the electronic properties of graphite was investigated theoretically and possible structural configurations were reported $[35,36]$. The first experimental realization of fluorinated graphene was done by Rahul et al. and it was reported that fluorographene is an insulator and it is stable up to $400^{\circ} \mathrm{C}$ [37]. Following the experiment, Sahin et al. investigated different configurations of fluorines on graphene and showed that different structures of fluorinated graphene can be formed at different levels of $F$ coverage which are all dynamically stable [38]. Later, Yang et al. investigated the Raman spectroscopy of fluorinated graphene structures for a different number of layers [39] and found that monolayer graphene is much more susceptible to fluorination. In addition, it was reported that the fluorination of $N$-layer graphene is reversible after vacuum annealing.

Fully functionalized forms of some novel monolayers such as graphane and fluoro-graphene, have been considered in both experiments and theoretical studies due to enhanced properties of the monolayer structures. To our knowledge, the impact of full-surface fluorination on the properties of monolayer InSe have not been reported up to date. Here, we 

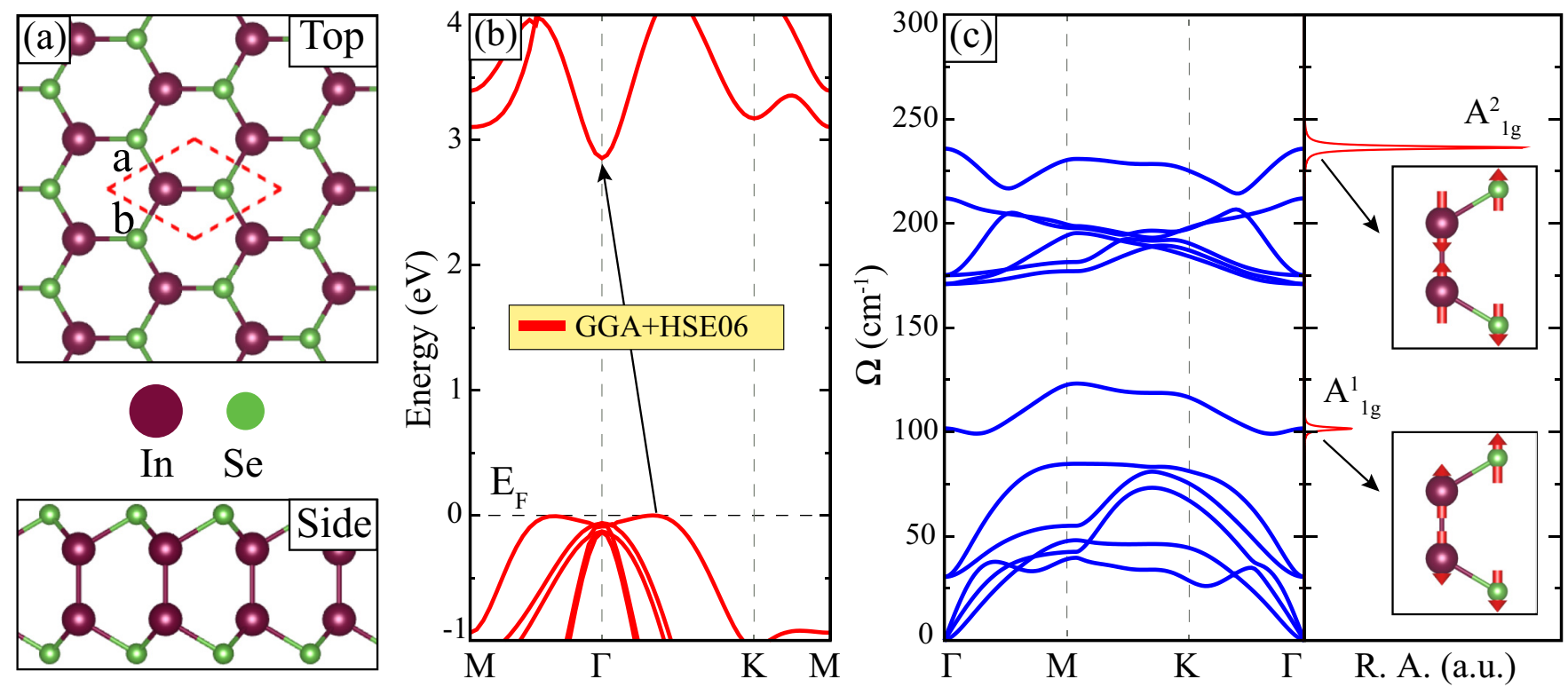

FIG. 1. For the monolayer InSe crystal, (a) top and side views of the structure, (b) electronic band dispersions, and (c) phonon band dispersions and corresponding Raman spectrum. The two prominent Raman active phonon modes are shown in the inset of (c).

predict the stability of a thin monolayer structure obtained by full-surface fluorination of InSe, namely monolayer InSeF. Our results reveal that the dynamically stable monolayer InSeF possesses distinctive structural, electronic, vibrational, and elastic properties as compared to monolayer InSe.

\section{COMPUTATIONAL METHODOLOGY}

For our first-principles calculations, we employed the plane-wave basis projector augmented wave (PAW) method in the framework of density-functional theory (DFT). The generalized gradient approximation (GGA) in the PerdewBurke-Ernzerhof (PBE) form [40,41] was employed for the exchange-correlation potential as implemented in the Vienna ab initio Simulation Package (VASP) $[42,43]$. The electronicband structure calculations were performed Heyd-ScuseriaErnzerhof (HSE06) [44] screened-nonlocal-exchange functional of the generalized Kohn-Sham scheme on top of GGA. The vdW correction to the GGA functional was included by using the DFT-D2 method of Grimme [45]. Analysis of the charge transfers in the structures was determined by the Bader technique [46].

Electronic and geometric relaxations of the monolayers of InSe and InSeF were performed on 4- and 6-atom unit cells, respectively. The kinetic energy cutoff for plane-wave expansion was set to $500 \mathrm{eV}$ and the energy was minimized until its variation in the following steps became $10^{-8} \mathrm{eV}$. The Gaussian smearing method was employed for the total energy calculations and the width of the smearing was chosen as $0.05 \mathrm{eV}$. Total Hellmann-Feynman forces in the primitive unitcell was reduced to $10^{-7} \mathrm{eV} / \AA$ for the structural optimization. $21 \times 21 \times 1 \Gamma$ centered $k$-point sampling was used for the primitive unit cells. To avoid interaction between the neighboring layers, a vacuum space of $25 \AA$ was implemented in the calculations. Phonon dispersion curves were calculated by using the small displacement method as implemented in the PHON code [47]. The phonon frequencies and the corresponding off-resonant Raman activities were calculated at the $\Gamma$ point of the Brillouin zone (BZ) by using the same methodology. A detailed theoretical explanation can be found in our previous reports $[48,49]$.

\section{MONOLAYER InSe}

Crystal structure of monolayer InSe consists of 2-Indium layers sandwiched between Se layers in the Se-In-In-Se order [see Fig. 1(a)]. For the rest of the paper we define the monolayer InSe in such a way that it is formed by the covalent bonding of two In-Se layers. The lattice constants $(a=b)$ are calculated to be $3.94 \AA$ which is slightly larger than the other to Ga-monochalcogenides ( 3.58 and $3.75 \AA$ for monolayers of $\mathrm{GaS}$ and GaSe structures, respectively) [17] due to the larger atomic radius of the In atom. The In-In bond length is found to be $2.77 \AA$ while that of In-Se is slightly smaller $(2.65 \AA)$. The Bader charge analysis reveals that each In atom donates its $0.6 e$ to a Se atom indicating the mostly covalent character of the In-Se bonds. Moreover, the work function of monolayer InSe is found to be $5.70 \mathrm{eV}$ which is slightly smaller than that of $\mathrm{GaSe}(5.59 \mathrm{eV})$ [17] due to lower ionization energy of the In atom than that of the $\mathrm{Ga}$ atom.

The calculated electronic band structure of monolayer InSe is shown in Fig. 1(b). The valence band maximum (VBM) lies between the $\Gamma-\mathrm{K}$ points while the conduction band minimum (CBM) is located at the $\Gamma$ point in the BZ. The calculated indirect band gap is $2.85 \mathrm{eV}$ which is slightly smaller than the experimentally reported band gap $(\sim 2.9 \mathrm{eV})[6]$. Despite very close energies of two valence band edges, the monolayer InSe is certainly an indirect gap semiconductor due to its weak PL intensity [6].

As shown in Fig. 1(c), the monolayer InSe does not exhibit any imaginary frequency through the whole BZ suggesting its dynamical stability. As given by the first-order off-resonant 
Raman spectrum [on the right panel of Fig. 1(c)], the monolayer InSe exhibits two prominent Raman active modes which are attributed to the out-of-plane vibration of the In and Se atoms in two different configurations as shown in the inset of Fig. 1(c). The frequency of the $\mathrm{A}_{1 g}^{1}$ phonon mode is calculated to be $101.6 \mathrm{~cm}^{-1}$ and its Raman activity is smaller than that of $\mathrm{A}_{1 g}^{2}$. The $\mathrm{A}_{1 g}^{1}$ phonon mode demonstrates the breathing vibrations of each In-Se layer. Notably, the chemical bonding between In atoms from top and bottom layers results in a phonon hardening for the $\mathrm{A}_{1 g}^{1}$ phonon mode as compared to weakly interacting layers. On the other hand, the $\mathrm{A}_{1 g}^{2}$ phonon mode indicates the opposite out-of-plane vibration of In and Se atoms, respectively, and its frequency is found to be $236.4 \mathrm{~cm}^{-1}$. Apart from the two out-of-plane phonon modes, there are also three doubly degenerate in-plane Raman active modes which have much smaller Raman activities than those of prominent modes.

\section{INTERACTION OF MONOLAYER InSe WITH SINGLE FLUORINE}

The adsorption is an efficient way to tune the structural, electronic, and magnetic properties of 2D materials. Fluorine atom is a potential candidate for such functionalization with its relatively high electronegativity (3.98 with respect to the Pauling scale). Among 2D monolayer materials, adsorption of the $\mathrm{F}$ atom on graphene layer (fluoro-graphene) was theoretically predicted and experimentally demonstrated. In order to functionalize the monolayer InSe, first the interaction of a single $\mathrm{F}$ with the InSe surface is investigated on a 36-atom supercell. Four different adsorption sites, the top of the In atom $\left(\mathrm{T}_{\mathrm{In}}\right)$, that of the $\mathrm{Se}$ atom $\left(\mathrm{T}_{\mathrm{Se}}\right)$, bridge $(\mathrm{B})$, and hollow (H) sites [see Fig. 2(a)], are considered. Among these sites, $T_{\text {In }}$ is found to be the energetically most favorable site for the adsorption of an F atom. As listed in Table I, the corresponding binding energy of the $\mathrm{F}$ atom is calculated to be $2.38 \mathrm{eV}$ which is smaller but close to that of on graphene $(2.71 \mathrm{eV})$ [38]. The In-F bond length is found to be $2.04 \AA$ which is much longer than a typical C-F bond and other F bonds. Since the F atom has a high electronegativity, a remarkable amount of charge $(0.7 e)$ is donated from an In atom on which it is adsorbed. Strong interaction between the In and F atoms causes the In atom to be destroyed towards the F atom as seen in Fig. 2(c).

Adsorption of a single $\mathrm{F}$ atom on the monolayer InSe preserves the semiconducting nature of InSe with induced midgap states arising from In atoms [see Fig. 2(d)]. Single $\mathrm{F}$ adsorption on the monolayer InSe introduces a magnetic ground state with a local magnetic moment of $1 \mu_{B}$. As seen in Fig. 2(b), when a single F atom is bound to an In atom, the inversion symmetry of the monolayer $\operatorname{InSe}(z \rightarrow-z)$ is broken and the lower In atom has unoccupied $p_{z}$ orbitals which results in the magnetic ground state. Notably, as discussed in the case of graphene that most of the adsorbed atoms on graphene layer induces a local magnetic moment of $1 \mu_{B}$ which is universal and created by direct bonding between the adatom and $\mathrm{C}$ atom [50,51]. As it was reported, the F atom is such a candidate for the creation of such a local magnetic moment due to its strong bonding on the $\mathrm{C}$ site resulting in $s p^{3}$-type hybridization. A similar situation holds for the monolayer InSe when a single (a)
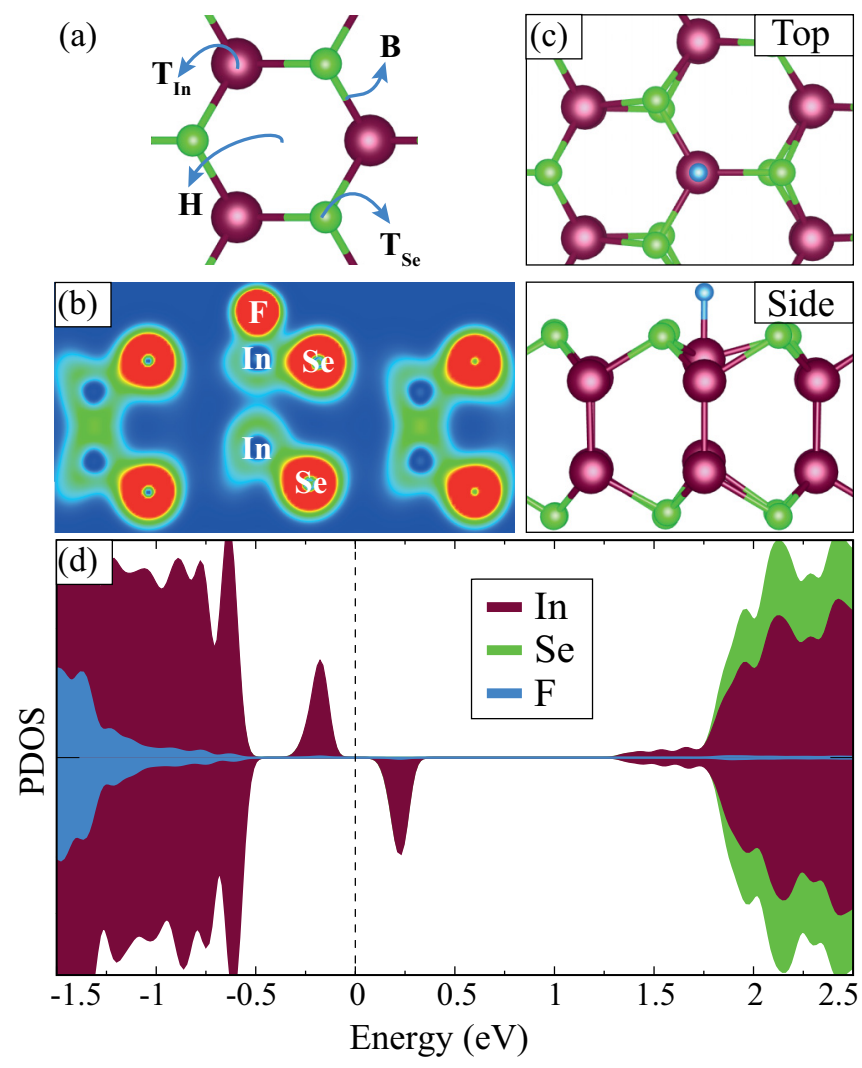

FIG. 2. (a) Schematic representation of the possible adsorption sites for the single $\mathrm{F}$ atom. (b) Increasing charge density is shown by a color scheme from blue to red with linear scaling between zero (blue) and $7.7 e / \AA^{3}$ (red). (c) Top and side views of the single $\mathrm{F}$ adsorbed monolayer InSe. (d) The corresponding partial density of states (PDOS).

$\mathrm{F}$ atom is adsorbed on top of an In atom and the induced In-F bonding destroys the hybridization in the InSe structure. In addition, the analysis of partial density of states shows the contribution of In orbitals to the net magnetic moment in the single F-adsorbed monolayer InSe [see Fig. 2(d)].

TABLE I. For the adsorption of a single $\mathrm{F}$ atom on the monolayer InSe, calculated vertical distance of the F atom to the surface $h$, the bond length between $\mathrm{F}$ and In atoms $d_{F-I n}$, the amount of donated charge from the In atom to the $\mathrm{F}$ atom $\Delta \rho(\mathrm{In}-\mathrm{F})$, that to the Se atom $\Delta \rho(\mathrm{In}-\mathrm{Se})$, the amount of donated charge from the Se atom to the $\mathrm{F}$ atom $\Delta \rho(\mathrm{Se}-\mathrm{F})$, the local magnetic moment of the F-adsorbed monolayer InSe $\mu$, and binding energy of the $\mathrm{F}$ atom to the surface $E_{b}$.

\begin{tabular}{lccccccc}
\hline \hline $\begin{array}{l}\text { Binding } \\
\text { site }\end{array}$ & $h$ & $d_{\mathrm{F}-\mathrm{In}}$ & $\begin{array}{c}\Delta \rho \\
(\mathrm{In}-\mathrm{F})\end{array}$ & $\begin{array}{c}\Delta \rho \\
(\mathrm{In}-\mathrm{Se}) \\
(e)\end{array}$ & $\begin{array}{c}\Delta \rho \\
(\mathrm{Se}-\mathrm{F}) \\
(e)\end{array}$ & $\left(\mu_{B}\right)$ & $(\mathrm{eV})$ \\
\hline $\mathrm{T}_{\mathrm{In}}$ & 2.10 & 2.04 & 0.7 & 0.6 & - & 1.0 & 2.38 \\
$\mathrm{~T}_{\mathrm{Se}}$ & 1.90 & - & - & 0.6 & - & 1.0 & 2.06 \\
$\mathrm{H}$ & 1.25 & - & - & 0.6 & - & 1.0 & 2.09 \\
$\mathrm{~B}$ & 1.63 & - & - & 0.6 & 0.6 & 1.0 & 2.14 \\
\hline \hline
\end{tabular}



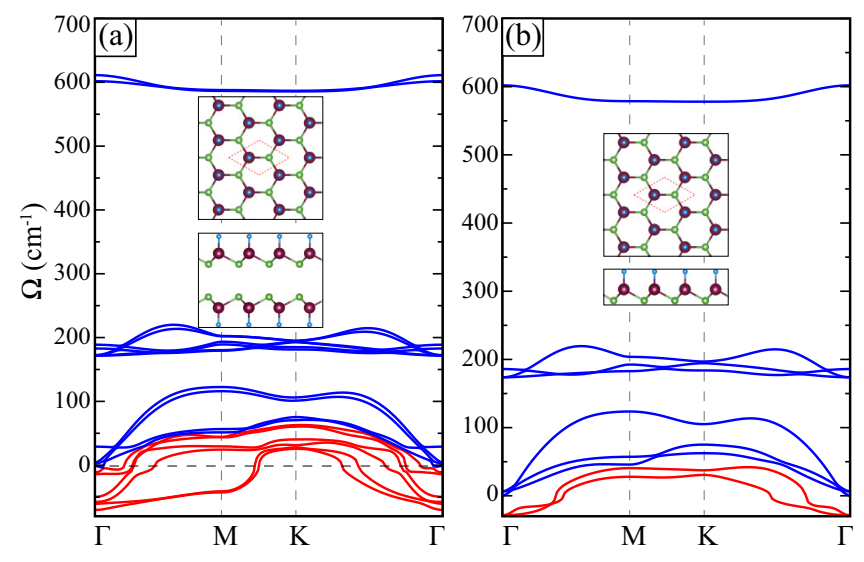

FIG. 3. Calculated phonon-band structures of (a) fully fluorinated monolayer InSe and (b) In-Se-F layer. The insets show the top and side views of the crystal structures. The red solid lines represent the phonon branches which display imaginary frequencies in the BZ.

\section{FULL FLUORINATION OF MONOLAYER InSe}

Following the analysis of the interaction of the single $\mathrm{F}$ atom on the monolayer InSe, we investigate the effect of full fluorination of the monolayer InSe on its properties. As mentioned in Sec. IV, energetically the most favorable site is the top of the In atom for the adsorption of a F atom. Thus, for full-surface fluorination, we systematically saturate each In atom with a $\mathrm{F}$ atom. The optimized geometry reveals that In and $\mathrm{F}$ atoms tend to form In-F pairs which are linked by $\mathrm{Se}$ atoms to form a hexagonal $1 \mathrm{H}$ structure. It is clear that the strong In-In dimer bonds are broken by the adsorption of $\mathrm{F}$ atoms on In sites. In the final structure, two weakly coupled In-Se-F layers are formed which are found to be dynamically unstable. Moreover, an isolated layer of In-Se-F compositions is also found to possess dynamical instability [see Figs. 3(a) and $3(b)]$.

As shown in Fig. 3(a), four phonon branches display mostly imaginary frequencies through the whole BZ indicating its dynamical instability. Three of these phonon branches are acoustical phonons while the other one is attributed to the in-plane shear vibration of each In-Se-F layer. The latter mode suggests that the two In-Se-F layers are detached and the interlayer interaction (only $30 \mathrm{meV} /$ layer in this case) is even much weaker than a typical vdW interaction. Therefore, it is concluded that direct fluorination of monolayer InSe through In sites from each surface cannot reveal a dynamically stable In-Se-F composition. For this reason, we consider other fluorination configurations in which the In-Se layer is fluorinated from both surfaces.

\section{Stable monolayer InSeF}

\section{Structural and electronic properties}

As mentioned above, dynamically unstable fluorinated monolayer InSe can be stabilized upon changing the fluorination configuration of the In-Se-F layers. Instead of the crystal structure shown in the inset of Fig. 3(b), when a F atom binds to an In atom from the top and another F binds to the nearest In atom from the bottom surface (like the structure of graphane),
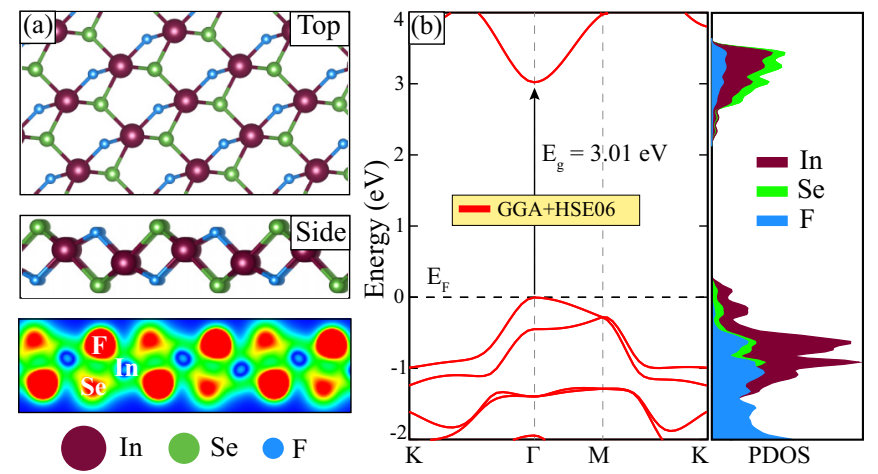

FIG. 4. For the monolayer InSeF, (a) top and side views and the corresponding charge distribution from side view. Increasing charge density is shown by a color scheme from blue to red with linear scaling between zero (blue) and $7.7 e^{-} / \AA^{3}$ (red). (b) The electronic band dispersions and the corresponding partial density of states (PDOS). The Fermi level is set to zero.

the In-Se-F layer is dynamically stabilized. For the rest of the paper, we define this stable structure as the monolayer InSeF. For this configuration, a $2 \times 1 \times 1$ supercell is constructed [see Fig. 4(a)]. The optimized crystal structure possesses a distorted, anisotropic 1T-like phase in which each In atom is five-coordinated with two $\mathrm{F}$ and three Se atoms. In addition, each 1D In chain is linked by Se atoms. The optimized lattice parameters for the monolayer InSeF are calculated to be $a=6.44 \AA$ and $b=3.84 \AA$ indicating the anisotropy of the primitive unit cell. The In-Se bond length is calculated to be $2.63 \AA$ in the chain and $2.68 \AA$ A between the chains. In addition, the In-F bond length is found to be $2.23 \AA$ which is larger than that in the single F-adsorbed structure (2.04 $\AA$ ). The thickness of the monolayer InSeF is $3.08 \AA$ which is much thinner than the monolayer InSe (5.50 A). According to the Bader charge analysis, it is found that each In atom donates its $0.5 e$ to a Se atom and $0.7 e$ to a $\mathrm{F}$ atom due to larger electron affinity of the $\mathrm{F}$ atom [see bottom panel of Fig. 4(a)]. In addition, the work function of the monolayer InSeF $(6.28 \mathrm{eV})$ is calculated to be larger than that of the monolayer InSe. This can be attributed to the formation of rather strong In-F bonds on both surfaces.

The electronic band structure and the corresponding PDOS of the monolayer InSeF are presented in Fig. 4(b). As shown by the PDOS, F atoms mostly contribute to valence band states while the $\mathrm{Se}$ atoms contribute to both valence and conduction bands. However, the few top valence bands are dominated by the In atoms. It appears from the electronic band dispersions that in contrast to the monolayer InSe, the monolayer InSeF is a direct band gap semiconductor with a band gap of $3.01 \mathrm{eV}$ which is slightly larger than that of InSe $(2.85 \mathrm{eV})$. The VBM and CBM reside at the $\Gamma$ point. Moreover, it is seen that the bands are highly anisotropic around the $\Gamma$ point due to the existing 1D-like chains in the structure.

\section{Vibrational properties and Raman spectrum}

The dynamical stability of the monolayer InSeF is verified by calculating its phonon band dispersions through the whole BZ. As shown in Fig. 5(a), the phonon bands of the 


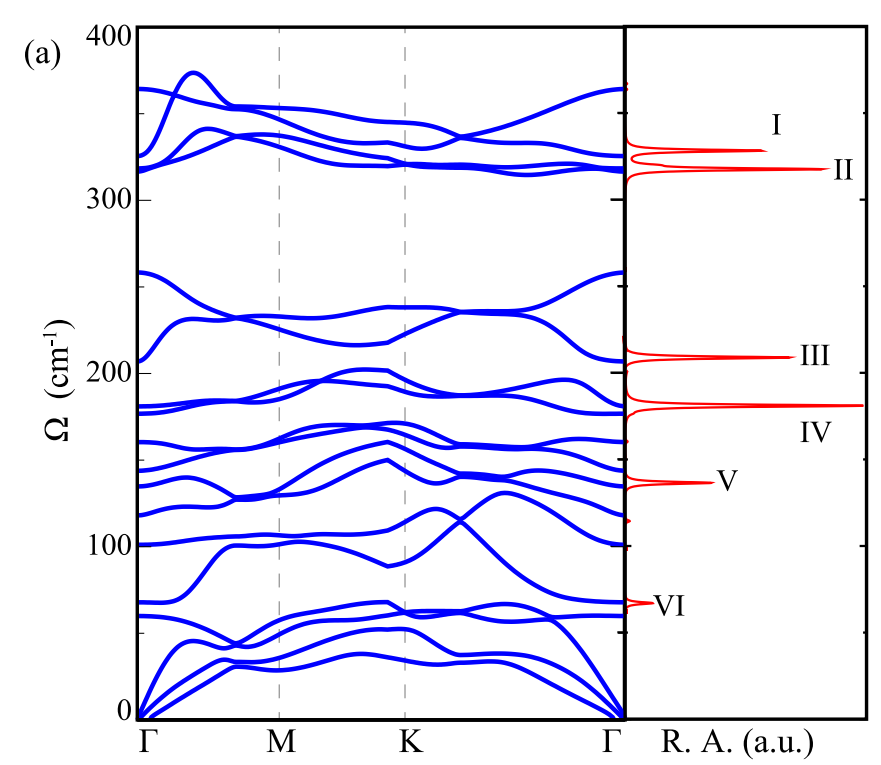

(b)

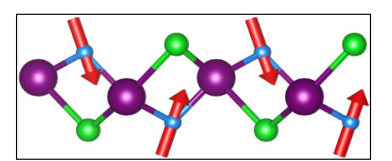

I $\left(325.0 \mathrm{~cm}^{-1}\right)$

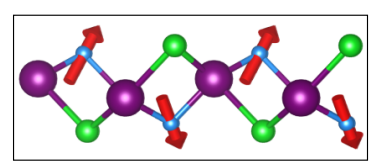

II $\left(314.3 \mathrm{~cm}^{-1}\right)$

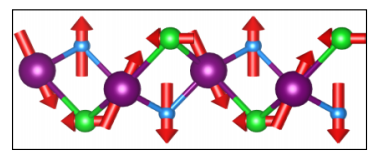

III $\left(206.9 \mathrm{~cm}^{-1}\right)$

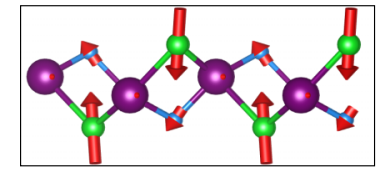

IV $\left(181.0 \mathrm{~cm}^{-1}\right)$

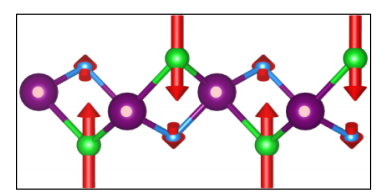

$\mathrm{V}\left(143.9 \mathrm{~cm}^{-1}\right)$

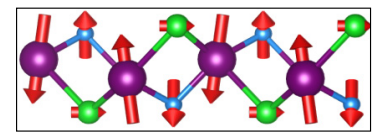

VI $\left(67.0 \mathrm{~cm}^{-1}\right)$

FIG. 5. (a) Calculated phonon-band structure and the corresponding Raman activities of the phonon modes for the monolayer InSeF. (b) Characteristic of six Raman-active modes with their frequencies.

monolayer InSeF are free from the imaginary frequencies in the whole BZ indicating the dynamical stability of the monolayer structure. There are 18 phonon branches three of which are acoustical. The highest-frequency optical phonon branch is calculated to be at $363.7 \mathrm{~cm}^{-1}$ which is much larger than that of the monolayer InSe $\left(235.9 \mathrm{~cm}^{-1}\right)$. The relatively high-frequency phonon modes appear due to In-F

bond stretching. As shown in Fig. 5(a), the calculated Raman spectrum of the monolayer InSeF exhibits more prominent Raman active phonon modes than that of the monolayer InSe. The Raman spectrum of the monolayer InSeF displays a large optical phonon gap which distinguishes the phonon modes arising from In-F bonds. As shown in Fig. 5(b), the two Raman active modes having frequencies 325.0 and $314.3 \mathrm{~cm}^{-1}$ are attributed to the opposite vibration of $\mathrm{F}$ atoms. In both phonon modes vibration of the $\mathrm{F}$ atoms are coupled motions along in-plane and out-of-plane directions. The other four prominent Raman active modes are found to have frequencies 206.9, 181.0, 143.9, and $67.0 \mathrm{~cm}^{-1}$. The phonon mode having frequency $206.9 \mathrm{~cm}^{-1}$ is attributed to the opposite vibration of In and $\mathrm{F}$ atoms in out-of-plane direction while Se atoms have small in-plane contribution. The Raman active mode at frequency $181.0 \mathrm{~cm}^{-1}$ is mostly dominated by the out-ofplane vibration of Se atoms against each other while $\mathrm{F}$ atoms have small coupled in-plane and out-of-plane contribution. Moreover, the phonon mode having frequency $143.9 \mathrm{~cm}^{-1}$ has nearly the same vibrational character, however, the Se-F pairs vibrate purely along the out-of-plane direction. The lowest frequency prominent phonon mode at frequency $67.0 \mathrm{~cm}^{-1}$ is attributed to the mostly out-of-plane vibration of In-F pairs against each other. As shown by the Raman spectrum, the monolayer InSeF displays significant distinctive features in its vibrational spectrum as compared to the monolayer InSe. Therefore, it can be concluded that the Raman spectrum of monolayers of InSe and InSeF are totally different so that the two structures are distinguishable in terms of their vibrational properties.

\section{Mechanical properties}

The linear-elastic properties of 2D monolayer materials can be represented in terms of two independent constants: the in-plane stiffness $C$, and the Poisson ratio $\nu$. For determination of linear-elastic constants, the relaxed-ion elastic tensors of monolayers InSe and InSeF are calculated by using smalldisplacement methodology. In order to obtain the anisotropy in the linear-elastic constants, the orientation angle-dependent $(\theta)$ elastic constants are calculated by using the equations [52]:

$$
\begin{aligned}
C(\theta) & =\frac{C_{11} C_{22}-C_{12}^{2}}{C_{22} \cos ^{4}(\theta)+A \cos ^{2}(\theta) \sin ^{2}(\theta)+C_{11} \sin ^{4}(\theta)}, \\
\nu(\theta) & =\frac{C_{12} \cos ^{4}(\theta)-B \cos ^{2}(\theta) \sin ^{2}(\theta)+C_{12} \sin ^{4}(\theta)}{C_{22} \cos ^{4}(\theta)+A \cos ^{2}(\theta) \sin ^{2}(\theta)+C_{11} \sin ^{4}(\theta)},
\end{aligned}
$$

TABLE II. For the monolayers of InSe and InSeF, optimized lattice parameters, $a$ and $b$, the thickness of the crystal structure $h$, the bond length between constituent atoms $d_{X-Y}$, the amount of donated charge $\Delta_{\rho(X-Y)}$, the magnetic state MS, energy band gap calculated within HSE on top of GGA $E_{\mathrm{gap}}^{\mathrm{GG}+\mathrm{HSE}}, \mathrm{VBM}$ and CBM positions, the work function calculated from the F-adsorbed surface $\Phi$, and linear-elastic

\begin{tabular}{|c|c|c|c|c|c|c|c|c|c|c|c|c|c|c|}
\hline & $\begin{array}{c}a \\
(\AA)\end{array}$ & $\begin{array}{c}b \\
(\AA)\end{array}$ & $\begin{array}{c}h \\
(\AA)\end{array}$ & $\begin{array}{c}d_{\mathrm{In}-\mathrm{Se}} \\
(\AA)\end{array}$ & $\begin{array}{c}d_{\mathrm{In}-\mathrm{F}} \\
(\AA)\end{array}$ & $\begin{array}{c}\Delta_{\rho(\mathrm{In}-\mathrm{Se})} \\
(e)\end{array}$ & $\begin{array}{c}\Delta \rho_{(\mathrm{In}-\mathrm{F})} \\
(e)\end{array}$ & $\begin{array}{l}\text { MS } \\
(-)\end{array}$ & $\begin{array}{c}E_{\mathrm{gap}}^{\mathrm{GGA}+\mathrm{HSE}} \\
(\mathrm{eV})\end{array}$ & VBM/CBM & $\begin{array}{c}\Phi \\
(\mathrm{eV})\end{array}$ & $\begin{array}{c}\mathrm{C}_{11} \\
(\mathrm{~N} / \mathrm{m})\end{array}$ & $\begin{array}{c}\mathrm{C}_{22} \\
(\mathrm{~N} / \mathrm{m})\end{array}$ & $\begin{array}{c}\mathrm{C}_{12} \\
(\mathrm{~N} / \mathrm{m})\end{array}$ \\
\hline $\mathrm{InSe}$ & 3.94 & 3.94 & 5.50 & 2.65 & - & 0.6 & - & NM & 2.85 & $\Gamma-\mathrm{K} / \Gamma$ & 5.70 & 54.4 & 54.4 & 16.2 \\
\hline
\end{tabular}
coefficients $C_{i j}$. 
(a) $\mathrm{C}(\theta)$ in $\mathrm{N} / \mathrm{m} \quad 90^{\circ}$

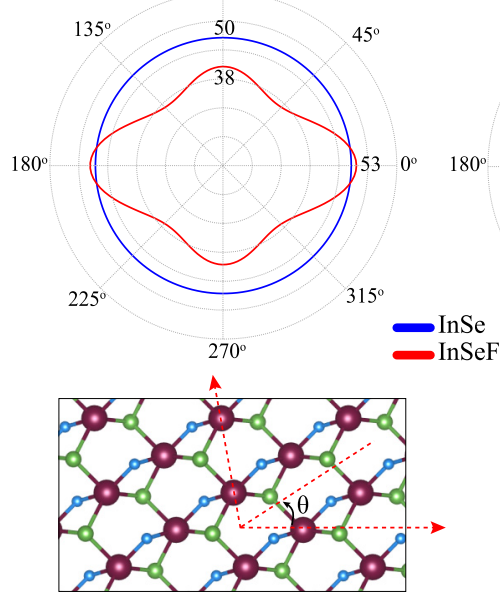

(b) $v(\theta)$

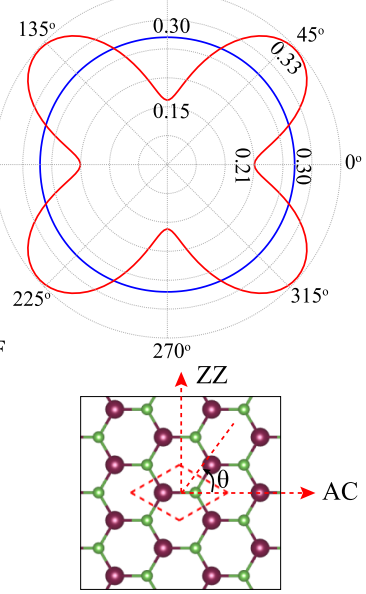

FIG. 6. For the monolayers of InSe and InSeF: (a) the orientation angle-dependent in-plane stiffness and (b) that of the Poisson ratio. The angle $\theta$ is defined for both monolayers as shown on the bottom panel.

where the constants $A$ and $B$ are defined such that $A=$ $\left(C_{11} C_{22}-C_{12}^{2}\right) / C_{66}-2 C_{12}$ and $B=C_{11}+C_{22}-\left(C_{11} C_{22}-\right.$ $\left.C_{12}^{2}\right) / C_{66}$.

First, by using the linear-elastic coefficients $C_{i j}$, the mechanical stability of the monolayer $\mathrm{InSeF}$ is verified. It is known that for a mechanically stable 2D monolayer material, the elastic coefficients should obey the Born criteria [53]: $C_{11} C_{22}-C_{12}^{2}>0$ which is satisfied for the monolayer InSeF (the values of $C_{i j}$ are listed in Table II). For the mechanically stable monolayer InSeF, we also investigate the orientation angle-dependent in-plane stiffness and Poisson ratio by using Eqs. (1) and (2), respectively. As shown in Fig. 6, the monolayer InSe exhibits totally in-plane isotropy behavior due to its symmetric hexagonal lattice. The calculated in-plane stiffness values are $50 \mathrm{~N} / \mathrm{m}$ along $\mathrm{ZZ}$ and $\mathrm{AC}$ directions, respectively. In contrast, the monolayer InSeF displays significant in-plane anisotropy because of the 1D-like chains in its crystal structure. According to the defined orientation angle in Fig. 6, the in-plane stiffness along the 1D chain (parallel to chain) is calculated to be $43.8 \mathrm{~N} / \mathrm{m}$ while it is found slightly smaller perpendicular to the chains $(38.5 \mathrm{~N} / \mathrm{m})$. This slight difference can be attributed to the relatively larger distance between the $1 \mathrm{D}$ chains. In addition, apart from these two main orienta- tions, the monolayer InSeF possesses slightly larger in-plane stiffness $(53.0 \mathrm{~N} / \mathrm{m})$ at $\theta=0^{\circ}$ than that of the monolayer InSe. In the case of the Poisson ratio, the monolayer InSe has a value of 0.30 which is isotropic along any direction. On the other hand, the monolayer InSeF displays a strong orientation-angle dependence in terms of the Poisson ratio. The Poisson ratio is calculated to be 0.25 and 0.15 parallel and perpendicular to the $1 \mathrm{D}$ chains, respectively. The highly anisotropic nature of the monolayer InSeF makes it a suitable candidate for nanomechanical applications.

\section{CONCLUSION}

In this study, the formation of a thin monolayer structure, namely InSeF, via fluorination of the monolayer InSe was predicted by means of $a b$ initio calculations. It was revealed that strong interaction of $\mathrm{F}$ and In atoms leads to the detachment of In-Se layers, which are dynamically unstable, in the monolayer InSe. Further investigation of the different fluorination scenario in the isolated In-Se-F layer revealed the formation of the 1T-like monolayer InSeF structure. The monolayer InSeF was shown to exhibit dynamical stability in terms of its phonon band dispersions. In addition, its Raman spectrum was found to exhibit totally distinctive features as compared to the monolayer InSe. The electronic band structure calculations indicated that the highly anisotropic monolayer InSeF is a direct gap semiconductor with its valence and conduction band edges residing at the $\Gamma$ point. The orientation angledependent linear elastic constants of the monolayer InSeF indicated that it displays strong in-plane anisotropy in elastic constants and it is a slightly softer material as compared to the monolayer InSe. Overall, it was predicted that a thin, direct gap semiconducting monolayer structure can be formed by the full-fluorination monolayer InSe as a new member of the 2D family.

\section{ACKNOWLEDGMENTS}

Computational resources were provided by TUBITAK ULAKBIM, High Performance and Grid Computing Center (TR-Grid e-Infrastructure). This work is supported by the Flemish Science Foundation (FWO-V1) by a postdoctoral fellowship (M.Y.).
[1] K. S. Novoselov, A. K. Geim, S. V. Morozov, D. Jiang, Y. Zhang, S. V. Dubonos, I. V. Grigorieva, and A. A. Firsov, Science 306, 666 (2004).

[2] D. J. Late, B. Liu, J. Luo, A. Yan, H. S. S. R. Matte, M. Grayson, C. N. R. Rao, and V. P. Dravid, Adv. Mater 24, 3549 (2012).

[3] X. Li, M. W. Lin, A. A. Puretzky, J. C. Idrobo, C. Ma, M. Chi, M. Yoon, C. M. Rouleau, I. I. Kravchenko, D. B. Geohegan, and K. Xiao, Sci. Rep. 4, 5497 (2014).

[4] H. Cai, J. Kang, H. Sahin, B. Chen, A. Suslu, K. Wu, F. M. Peeters, X. Meng, and S. Tongay, Nanotech. 27, 065203 (2016).

[5] P. Hu, J. Zhang, M. Yoon, X. F. Qiao, X. Zhang, W. Feng, P. Tan, W. Zheng, J. Liu, X. Wang, J. C. Idrobo, D. B. Geohegan, and K. Xiao, Nano Res. 7, 694 (2014).
[6] D. A. Bandurin, A. V. Tyurnina, G. L. Yu, A. Mishchenko, V. Zólyomi, S. V. Morozov, R. K. Kumar, R. V. Gorbachev, Z. R. Kudrynskyi, S. Pezzini, Z. D. Kovalyuk, U. Zeitler, K. S. Novoselov, A. Patanè, L. Eaves, I. V. Grigorieva, V. I. Fal'ko, A. K. Geim, and Y. Cao, Nat. Nanotechnol. 12, 223 (2017).

[7] C. Tatsuyama, Y. Watanabe, C. Hamaguchi, and J. Nakai, J. Phys. Soc. Jpn. 29, 150 (1970).

[8] A. Yamamoto, A. Syouji, T. Goto, E. Kulatov, K. Ohno, Y. Kawazoe, K. Uchida, and N. Miura, Phys. Rev. B 64, 035210 (2001).

[9] M. Xu, T. Liang, M. Shi, and H. Chen, Chem. Rev. 113, 3766 (2013). 
[10] D. J. Late, B. Liu, H. S. S. R. Matte, C. N. R. Rao, and V. P. David, Adv. Func. Mater. 22, 1894 (2012).

[11] C. H. Ho, M. H. Hsieh, and C. C. Wu, Rev. Sci. Instrum. 77, 113102 (2006).

[12] T. Aono, K. Kase, and A. Kinoshita, J. Appl. Phys. 74, 2818 (1993).

[13] P. Hu, Z. Wen, L. Wang, P. Tan, and K. Xiao, ACS Nano 6, 5988 (2012).

[14] H. Chen, Y. Li, L. Huang, and J. Li, J. Phys. Chem. C 119, 29148 (2015).

[15] H. Chen, Y. Li, L. Huang, and J. Li, RSC Adv. 5, 50883 (2015).

[16] X. Zhou, J. Cheng, Y. Zhou, T. Cao, H. Hong, Z. Liao, S. Wu, H. Peng, K. Liu, and D. Yu, J. Am. Chem. Soc. 137, 7994 (2015).

[17] M. Yagmurcukardes, R. T. Senger, F. M. Peeters, and H. Sahin, Phys. Rev. B 94, 245407 (2016).

[18] S. Popovic, A. Tonejc, B. Grzeta-Plenkovic, B. Celustka, and R. Trojko, J. Appl. Cryst. 12, 416 (1979).

[19] A. Pfitzner and H. D. Lutz, J. Solid State Chem. 124, 305 (1996).

[20] A. Likforman, D. Carre, and R. Hillel, Acta Crystallogr. Sect. B. 34, 1 (1978).

[21] J. Ye, S. Soeda, Y. Nakamura, and O. Nittono, Jpn. J. Appl. Phys. 37, 4264 (1998).

[22] G. Han, Z. Chen, J. Drennan, and J. Zou, Small 10, 2747 (2014).

[23] G. W. Mudd, S. A. Svatek, T. Ren, A. Patane, O. Makarovsky, L. Eaves, P. H. Beton, Z. D. Kovalyuk, G. V. Lashkarev, Z. R. Kudrynskyi, and A. I. Dmitriev, Adv. Mater. 25, 5714 (2013).

[24] G. W. Mudd, A. Patane, Z. R. Kudrynskyi, M. W. Fay, O. Makarovsky, L. Eaves, Z. D. Kovalyuk, V. Zolyomi, and V. Falko, Appl. Phys. Lett. 105, 221909 (2014).

[25] G. W. Mudd, S. A. Svatek, L. Hague, O. Makarovsky, Z. R. Kudrynskyi, C. J. Mellor, P. H. Beton, L. Eaves, K. S. Novoselov, Z. D. Kovalyuk, E. E. Vdovin, A. J. Marsden, N. R. Wilson, and A. Patane, Adv. Mater. 27, 3760 (2015).

[26] S. R. Tamalampudi, Y.-Y. Lu, R. Kumar U., R. Sankar, C.-D. Liao, K. Moorthy B., C.-H. Cheng, F. C. Chou, and Y.-T. Chen, Nano Lett. 14, 2800 (2014).

[27] N. Balakrishnan, Z. R. Kudrynskyi, M. W. Fay, G. W. Mudd, S. A. Svatek, O. Makarovsky, Z. D. Kovalyuk, L. Eaves, P. H. Beton, and A. Patane, Adv. Opt. Mater. 2, 1064 (2014).

[28] D. T. Do, S. D. Mahanti, and C. W. Lai, Sci. Rep. 5, 17044 (2015).

[29] A. Kandemir and H. Sahin, Phys. Rev. B 97, 155410 (2018).

[30] E. Bringuier, A. Bourdon, N. Piccioli, and A. Chevy, Phys. Rev. B 49, 16971 (1994).

[31] S. D. Jones, J. Zhang, C. E. Petoukhoff, M. K. L. Man, S. Lei, R. Vajtai, P. M. Ajayan, D. Talbayev, J. Madeo, and K. M. Dani, Sci. Rep. 6, 22620 (2016).
[32] S. Lei, L. Ge, S. Najmaei, A. George, R. Kappera, J. Lou, M. Chhowalla, H. Yamaguchi, G. Gupta, R. Vajtai, A. D. Mohite, and P. M. Ajayan, ACS Nano 8, 1263 (2014).

[33] G. W. Mudd, M. R. Molas, X. Chen, V. Zolyomi, K. Nogajewski, Z. R. Kudrynskyi, Z. D. Kovalyuk, G. Yusa, O. Makarovsky, L. Eaves, M. Potemski, V. I. Falko, and A. Patane, Sci. Rep. 6, 39619 (2016).

[34] J. O. Sofo, A. S. Chaudhari, and G. D. Barber, Phys. Rev. B 75, 153401 (2007); M. Yagmurcukardes, C. Bacaksiz, R. T. Senger, and H. Sahin, 2D Mater. 4, 035013 (2017).

[35] J.-C. Charlier, X. Gonze, and J.-P. Michenaud, Phys. Rev. B 47, 16162 (1993).

[36] Y. Takagi and K. Kusakabe, Phys. Rev. B 65, 121103(R) (2002).

[37] R. R. Nair, W. Ren, R. Jalil, I. Riaz, V. G. Kravets, L. Britnell, P. Blake, F. Schedin, A. S. Mayorov, S. Yuan, M. I. Katsnelson, H.-M. Cheng, W. Strupinski, L. G. Bulusheva, A. V. Okotrub, I. V. Grigorieva, A. N. Grigorenko, K. S. Novoselov, and A. K. Geim, Small 6, 2877 (2010).

[38] H. Sahin, M. Topsakal, and S. Ciraci, Phys. Rev. B 83, 115432 (2011).

[39] H. Yang, M. Chen, H. Zhou, C. Qiu, L. Hu, F. Yu, W. Chu, S. Sun, and L. Sun, J. Phys. Chem. C 115, 16844 (2011).

[40] J. P. Perdew, K. Burke, and M. Ernzerhof, Phys. Rev. Lett. 77, 3865 (1996).

[41] J. P. Perdew, K. Burke, and M. Ernzerhof, Phys. Rev. Lett. 78, 1396 (1997).

[42] G. Kresse and J. Hafner, Phys. Rev. B 47, 558 (1993).

[43] G. Kresse and J. Hafner, Phys. Rev. B 49, 14251 (1994).

[44] J. Heyd, G. E. Scuseria, and M. Ernzerhof, J. Chem. Phys. 118, 8207 (2003).

[45] S. J. Grimme, Comput. Chem. 27, 1787 (2006).

[46] G. Henkelman, A. Arnaldsson, and H. Jonsson, Comput. Mater. Sci. 36, 354 (2006).

[47] D. Alfe, Comput. Phys. Commun. 180, 2622 (2009).

[48] M. Yagmurcukardes, F. M. Peeters, and H. Sahin, Phys. Rev. B 98, 085431 (2018).

[49] M. Yagmurcukardes, C. Bacaksiz, E. Unsal, B. Akbali, R. T. Senger, and H. Sahin, Phys. Rev. B 97, 115427 (2018).

[50] E. J. G. Santos, A. Ayuela, and D. Sanchez-Portal, New J. Phys. 14, 043022 (2012).

[51] W. Han, R. K. Kawakami, M. Gmitra, and J. Fabian, Nat. Nanotechnol. 9, 794 (2014).

[52] E. Cadelano, P. L. Palla, S. Giordano, and L. Colombo, Phys. Rev. B 82, 235414 (2010).

[53] J. Wang, S. Yip, S. R. Phillpot, and D. Wolf, Phys. Rev. Lett. 71, 4182 (1993). 